Average length of hospital stay in the last 30 days of life fell, equating to a saving of 220 bed days or $£ 88000 /$ year

Patient/relative feedback was very positive.

Discussion The data suggests ESC is acceptable to and benefits patients. Fixed term funding is enabling the service to be expanded short term. Robust data collection and service analysis continue.

\section{A HOSPICE BASED RENAL SUPPORTIVE CARE SERVICE: AN EVALUATION OF THE FIRST 3 YEARS}

Jane Whitehurst, Sue Goodall, Ellie Hayter, Kevin Blackett. St Barnabas House

\subsection{6/spcare-2020-PCC.199}

Background St Barnabas House launched a Renal Supportive Care (RSC) Service in April 2016. This consists of a RSC Clinical Nurse Specialist (CNS), consultant support and joint working with the local secondary care renal team. Since 2016, 110 patients with advance renal disease (CKD 5) have been referred to our RSC service, approximately $3 \%$ of the hospice's total referrals each year from a baseline of $<1 \%$. We audited the service to measure impact and inform ongoing service development.

Methods We performed a retrospective audit of system 1 data and data prospectively collected by the RSC CNS for patients on the RSC caseload between April 2016 to August 2019. Data included referral source, reason, duration, outcome and information regarding intervention, advance care planning (ACP) and place of death.

Results 139 referrals were received for 110 patients. Mean duration of referral was 105 days range (1-1103). Referrals were made for symptom control (42\%), ACP (39\%), Psychosocial support (13\%), decision making support - dialysis versus conservative care $(6 \%)$ and stopping dialysis (2\%), patients imminently dying were referred to the non-disease specific CNS team. $49 \%$ of patients accessed another hospice service eg Day Hospice in addition to RSC CNS input. Faceto-face patient contacts occurred in patient's homes (83\%), outpatients (16\%) and hospital (1\%). 66 patients were discharged following a completed care episode, 29 of these were re-referred. 52 patients died, 38 (73\%) had their preferred place of death (PPD) recorded prior to death. In this group $1(3 \%)$ died in hospital. The rate of hospital death increased to $46 \%$ (6 out of 14 ) in patients without a documented PPD.

Conclusions The RSC service at StBH has increased access to specialist palliative care for people with advanced renal disease. Participation in ACP for these patients increased the likelihood of achieving a death outside of hospital.

\section{Survivorship and rehabilitation | poster 180}

\section{COST-EFFECTIVENESS OF COPD MANAGEMENT IN THE UK}

Mitri A Ng. King's College London

10.1136/spcare-2020-PCC.200

Introduction Chronic Obstructive Pulmonary Disease (COPD) is a long-term condition characterised by breathlessness. The
WHO has projected a $30 \%$ increase in total COPD deaths worldwide over the next decade. In 2012, COPD accounted for 29,776 deaths in the UK, and it costs the NHS over $£ 8,000,000$ annually.

Method This systematic review was conducted via searches on PubMed, using key words 'COPD', 'Management', 'Cost-effectiveness', 'exercise', 'QALY', 'UK' and 'Pulmonary Rehabilitation'. Studies with non-cost-effective interventions were removed. Studies using 'Quality Adjusted Life Years' and/or 'Incremental cost-effectiveness ratio' were considered for this review. Prices were adjusted for inflation.

Results Studies reviewed suggest that current standard care is cost-effective by NICE standards ( $£ 20,000$ to $£ 30,000$ per QALY). Cost effectiveness was improved with the addition of roflumilast to ICS/LABA therapy, where adding roflumilast led to an ICER of $£ 24,976$ per QALY gained for severe and very severe COPD in 2018. Tiotropium (£1934-£2548 in 2018] per QALY) proved to be a superior treatment over ipratropium ( $£ 2256-£ 2973$ in 2018] per QALY) and salmeterol ( $£ 2143-£ 2823$ in 2018] per QALY). Umeclidinium bromide added onto ICS/LABA therapy led to an ICER of $£ 1310$ per QALY, an improvement over Tiotropium. Adding pulmonary rehabilitation to treatment for COPD was likely to result in financial benefits, with mean incremental cost of adding PR was $-£ 152$ (- $£ 200$ in 2018$)$. Physical activity improved costeffectiveness versus sedentary lifestyle, reducing exacerbations and increasing QALYs.

Conclusion Current medical management is well optimized with regards to price per QALY gained. Umeclidinium should be considered for treatment instead of tiotropium. Physical Activity reduces exacerbations, increases QALYs and improves cost-effectiveness. However, data on cost-effectiveness of PR for COPD were insufficient. Furthermore, cost-effectiveness data of medical treatment should be updated as drug prices may have changed.

\section{Symptom management | posters $181-202$}

\section{IMPLEMENTING PATIENT CENTRED GOALS INTO A BREATHLESSNESS SYMPTOM MANAGEMENT CLINIC}

Camilla Barrow, Ruth Roberts. Birmingham St Mary's Hospice

\subsection{6/spcare-2020-PCC.201}

Introduction Space to breathe is a 5 week programme, with a rehabilitative focus, launched in January 2019 and designed through a Breathlessness steering group. A literature review recommended the use of the Goal Attainment Score (GAS) (Brighton 2018) and this was included into holistic measures of the breathlessness service. GAS light was chosen to reduce user burden and form part of the assessment of the service, complementing the rest of the Outcome Assessment Complexity Collaborative (OACC) suite and Chronic respiratory Questionnaire (CRQ) and Medical Research Council score of breathlessness (MRC) scores improved.

Method The programme is based on the Cambridge Breathing Thinking Functioning approach (Cambridge University Hospitals NHS Foundation Trust 2019) and with a focus on mastery of symptoms of breathlessness. Sessions include taught educational sessions followed by group discussions and exercise. Participants were supported by carers and clinicians to set patient identified SMART goals. GAS light 
was undertaken in week 1 and 5 of a 5 week breathlessness programme.

Results A Total of 55 patients attended 161 session out of a potential 275 , resulting in combined total of 44 separate goals been set using the GAS Light template.

All goals were reviewed and were categorized from 3 options namely, 'a little better'; 'same' or 'worse'. 30 Goals were achieved with patients identifying them as 'a little better'. 15 goals were not achieved of those 11 remained the 'same' and 4 had got 'worse'.

23 patients did not set or complete goal setting activity due to not completing the course, declining to participate in goal setting or not returning/recording data. This is reflective of both the attendance figures and the challenges of implementing a new outcome measure in a MDT.

Conclusions Following a 5 week breathlessness programme some participants achieved and partially achieved goals important to them. Further evaluation is required.

\section{IMPROVEMENT IN PALLIATIVE CARE PRESCRIBING}

B Bonelli, D Monnery. The Clatterbridge Cancer Centre

\subsection{6/spcare-2020-PCC.202}

Background It was recognised that junior doctors in Clatterbridge Cancer Centre were unfamiliar with regional palliative care guidelines and therefore the management of common presentations of oncological and palliative care patients, resulting in delayed prescriptions, particularly out of hours.

The aim of the project was to improve knowledge of, and confidence with, the management of the most frequent palliative care issues and to improve the quality of prescribing prior to initial review by the palliative care team.

Methods Junior doctors completed a questionnaire to assess confidence in symptoms management. As baseline audit, palliative care referrals for December 2018 were reviewed retrospectively, with focus on: reasons for referral, actions carried out prior to referral and whether the interventions were appropriate and in accordance with regional guidance. These guidelines were made available on all computer desktops in March 2019, accompanied by training and communication at ward level to ensure awareness. For the post intervention measure, referrals in March 2019 were reviewed. Identical criteria were used to assess whether ready access to guidelines had improved initial patient management.

Results In December 2018, 31 out of 182 patients were referred to palliative care. Of these, 12 required review by palliative care directly for complex issues, inpatient follow-up, social/psychological support or because they were approaching end of life. Of the remaining 19 patients, $10(52.6 \%)$ had appropriate therapy initiated by junior doctors prior to palliative care review. In March 2019, 41 out of 169 patients were referred to palliative care. Of the 15 qualifying patients, 12 $(80 \%)$ were started on appropriate treatment. The percentage of referrals to palliative care for non-complex physical symptoms control dropped from $61 \%$ in December 2018 to $36 \%$ in March 2019.

Conclusions Overall, digital access to guidelines and training in their use resulted in an improvement in symptom management in inpatients.

\section{HOW ARE SPECIALIST PALLIATIVE CARE UNITS USING RANITIDINE IN THE MEDICAL MANAGEMENT OF ADULTS WITH MALIGNANT BOWEL OBSTRUCTION? A SURVEY OF UK HOSPICES}

Andrew Campbell, Fiona Rawlinson, Amy Gadoud. St Mary's Hospice (Ulverston), Cardiff University, Lancaster University

10.1136/spcare-2020-PCC.203

Background Malignant bowel obstruction (MBO) is a common presentation in palliative care and can cause challenging symptoms. It has been suggested that the H2-receptor antagonist ranitidine may have a role in the management of $\mathrm{MBO}$ as an antisecretory medication to reduce volume of gastrointestinal secretions.

Methods A questionnaire was sent via email to all UK adult hospices with inpatient units to investigate how ranitidine is currently being used for patients with MBO.

Results 60\% (99 of 165) of eligible hospices completed the questionnaire. $76 \%$ reported using hyoscine butylbromide most commonly as a first line antisecretory medication for patients with MBO.

$84 \%$ of hospices reported using ranitidine for selected patients although only $8 \%$ reported using it as a first line antisecretory agent. Hospices that use ranitidine are most commonly administering it via continuous subcutaneous infusion for patients with acid related symptoms (88\%), combined with other antisecretory medications (80\%), as a second or third line antisecretory medication (68\%) and for gastrointestinal protection (66\%). Reasons cited for not using ranitidine included a lack of perceived need, lack of evidence, non-inclusion in clinical guidelines and issues of practicality.

Responders highlighted the need for an individualised approach to prescribing in $\mathrm{MBO}$ with failure of other medications, nature of symptoms and nature of obstruction among factors influencing use of ranitidine.

Multiple hospices reported recently starting to use ranitidine or using it more frequently than they had done previously. An Australian randomised controlled trial that used ranitidine as part of a 'standard' treatment regime in $\mathrm{MBO}$ was a commonly cited instigator for change.

Conclusions Ranitidine is being used by the majority of UK hospices for selected adults with MBO despite a limited evidence base. Further research should be encouraged to evaluate the effectiveness of ranitidine and to clarify its role for patients with $\mathrm{MBO}$.

\section{COST-EFFECTIVENESS OF PULMONARY REHABILITATION: A SYSTEMATIC REVIEW}

Baldev Chahal, Deokhee Yi, Natasha Lovell, Irene J Higginson. King's College London

\subsection{6/spcare-2020-PCC.204}

Background Pulmonary rehabilitation (PR) is a complex intervention aimed at decreasing morbidity in patients with longterm lung conditions such as COPD, bronchiectasis and pulmonary fibrosis. In the NHS setting, it is an 8 week programme consisting of exercise training to reduce functional decline, and education to aid patients with self-management of their condition. Extensive evidence exists in support of the clinical effectiveness of PR but there is a paucity of studies evaluating the economics of PR. This review aimed to 